SUBJECT AREAS: REACTION MECHANISMS

RIBOSWITCHES

STATISTICAL PHYSICS

CONTROL THEORY

SCIENTIFIC REPORTS:

$3: 3458$

DOI: $10.1038 /$ srep03458

(2013)

Published:

10 December 2013

Updated: 11 July 2014

\section{CORRIGENDUM: Collective behaviours: from biochemical kinetics to electronic circuits}

\section{Elena Agliari, Adriano Barra, Raffaella Burioni, Aldo Di Biasio \& Guido Uguzzoni}

This Article contains an error in the order of the figures. Figure 4, Figure 5, and Figure 6 were published as Figure 6, Figure 4, and Figure 5 respectively. The figures of this Article appear in the correct order below, with their respective figure legends. In addition, the $\mathrm{x}$ axis of Figure 5 should be negative log $\alpha$. This is correct in Figure 5 below.

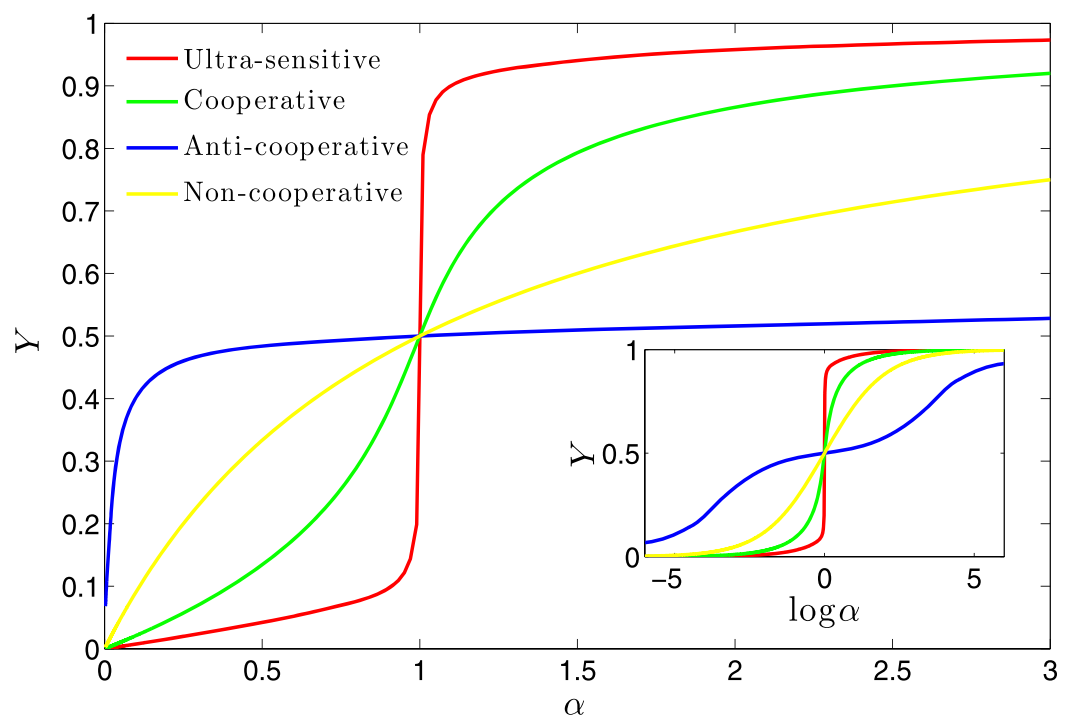

Figure $1 \mid$ Theoretical predictions of typical binding isotherms obtained from the statistical mechanics approach (see eq. 28) are shown versus the substrate concentration $\alpha$ (main plot) and versus the logarithm of the concentration (inset). Different colors refer to different systems (hence different coupling strengths $J$ ), as explained by the legend. In particular, as $J$ is varied, all the expected behaviors emerge: ultra-sensitive for $J=2$, cooperative for $J=1 / 2$, anti-cooperative for $J=-1 / 2$, non-cooperative for $J=0$. 

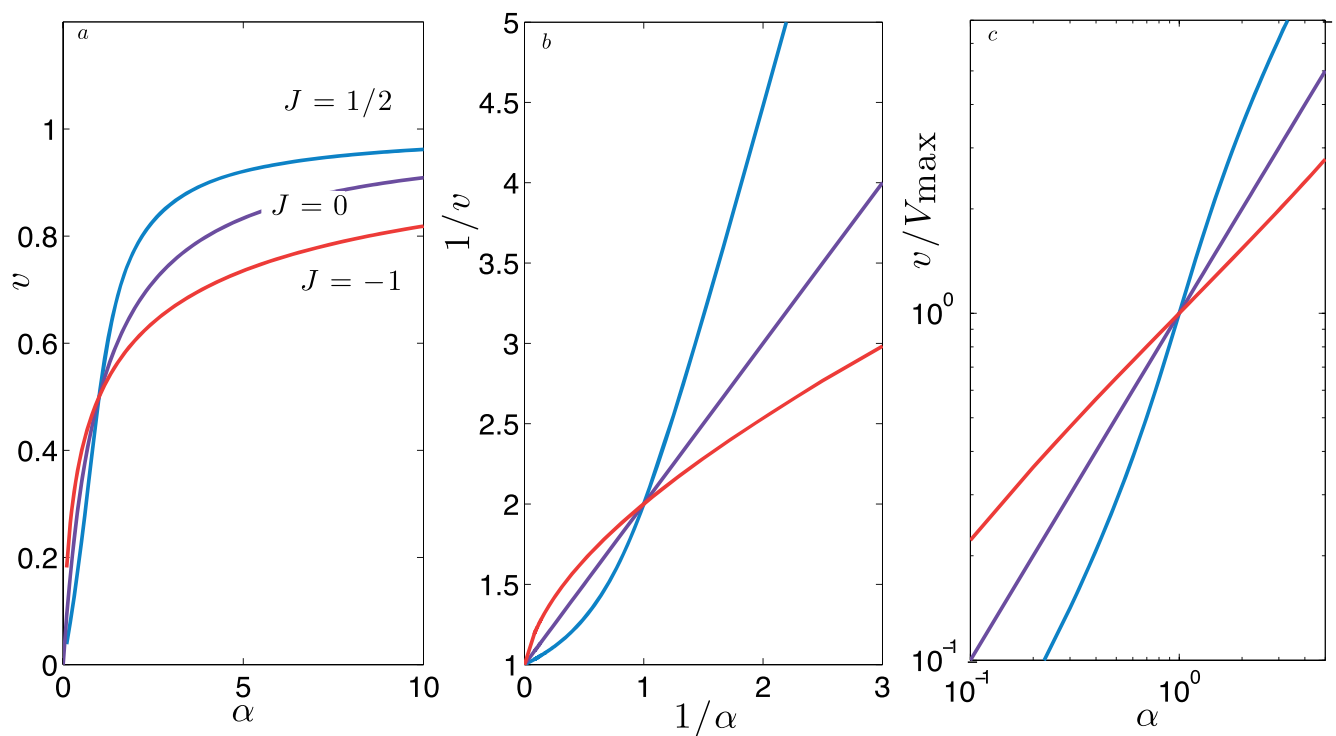

Figure $2 \mid$ Velocity of reactions versus substrate concentration $\alpha$. These plots have been included to show the full agreement between our theoretical outcomes and the results presented in the celebrated paper by Levitzki and Koshland (see fig. 4 in Ref. 50). Different values of $n_{H}=1 / 2,1,2$, corresponding to $J=-1,0,1 / 2$, are shown in different colors. Note that for this analysis there is complete proportionality between the reaction rates $v$ and the saturation curves $Y$ due to the law of mass action ${ }^{50}$.
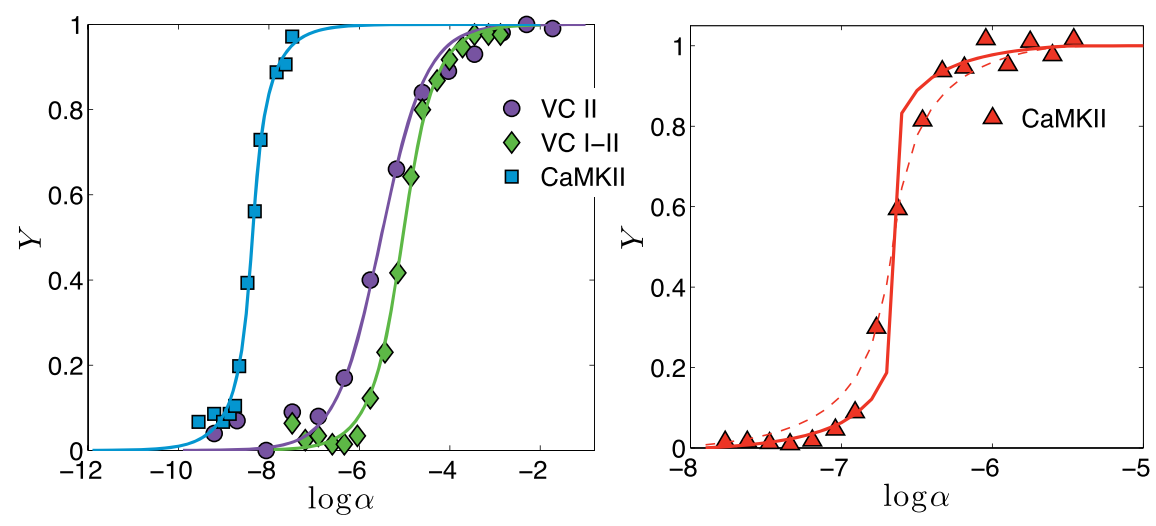

\begin{tabular}{cccc} 
data set & $J$ & $n_{H} \pm \Delta n_{H}$ & $n_{H}^{\text {lit }}$ \\
\hline \hline VC II & $0 \pm 0.6$ & $1.0 \pm 0.7$ & 0.97 \\
\hline VC I-II & $0.3 \pm 0.1$ & $1.4 \pm 0.2$ & 1.64 \\
\hline CaMKII & $0.67 \pm 0.03$ & $3.0 \pm 0.3$ & $3 \pm 0.3$
\end{tabular}

\begin{tabular}{ccc} 
data set & $J$ & $n_{H}^{\text {lit }}$ \\
\hline \hline CaMKII & $1.1 \pm 0.3$ & $4.4 \pm 0.6$
\end{tabular}

Figure 3 These plots show comparison between data from recent experiments (symbols) and best-fits through statistical mechanics (lines).

Data refer to non-cooperative and positive-cooperative systems ${ }^{22,51}$ (left panel) and an ultra-sensitive system ${ }^{52}$ (right panel). For the latter we report two fits: Dashed line is the result obtained by constraining the system to be cooperative but not-ultra-sensitive (that is, $J \leq 1$ ), while solid line is the best fit yielding $J \sim 1.1$, hence a "first order phase transition" in the language of statistical mechanics. The relative goodness of the fits are $\chi_{\text {coop }}^{2} \sim 0.85$ and $\chi_{\text {ultra }}^{2} \sim 0.94$, confirming an ultra-sensitive behavior. The tables in the bottom present the value of $J$ derived from the best fit and the resulting $n_{H}$; the estimate of the Hill coefficient taken from the literature is also shown. 


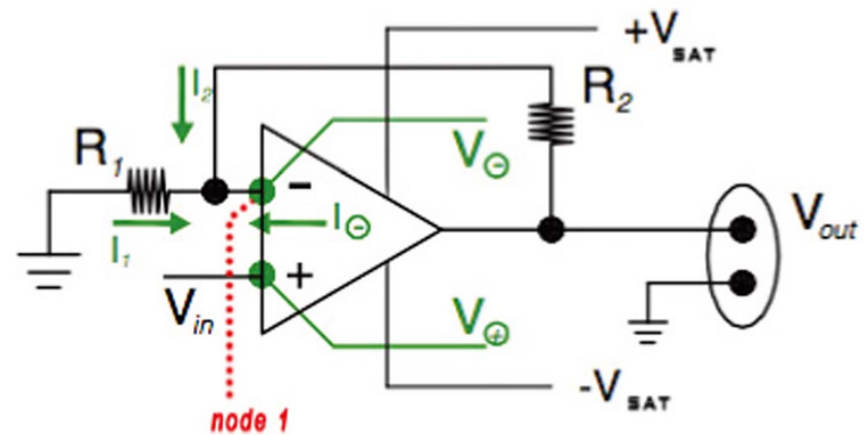

Figure 4 | Schematic representation of an operational amplifier.
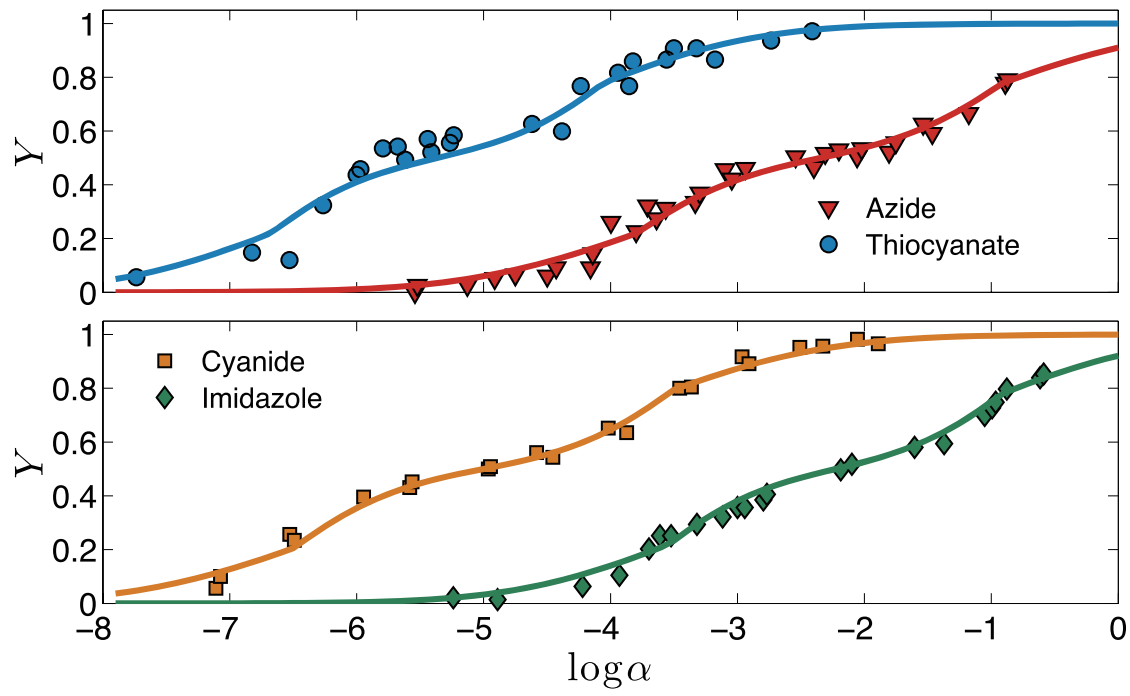

\begin{tabular}{cccc} 
data set & $J$ & $n_{H} \pm \Delta n_{H}$ & $n_{H}^{\text {fit }}$ \\
\hline \hline Azide & $3.0 \pm 0.1$ & $0.25 \pm 0.06$ & $0.23 \pm 0.06$ \\
\hline Thiocyanate & $2.5 \pm 0.3$ & $0.29 \pm 0.02$ & $0.28 \pm 0.06$ \\
\hline Cyanide & $3.0 \pm 0.1$ & $0.25 \pm 0.06$ & $0.22 \pm 0.05$ \\
\hline Imidazole & $2.7 \pm 0.4$ & $0.27 \pm 0.03$ & $0.25 \pm 0.05$
\end{tabular}

Figure $5 \mid$ Several sets of experimental data (symbols) ${ }^{53}$ are fitted by eq. 30 with minus sign (solid line). The values of $J$ corresponding to the best fits are shown in the table together with the related estimates for $n_{H}$ according to eq. 31 . The estimates for $n_{H}$ obtained via standard Hill fit are also shown. 
a
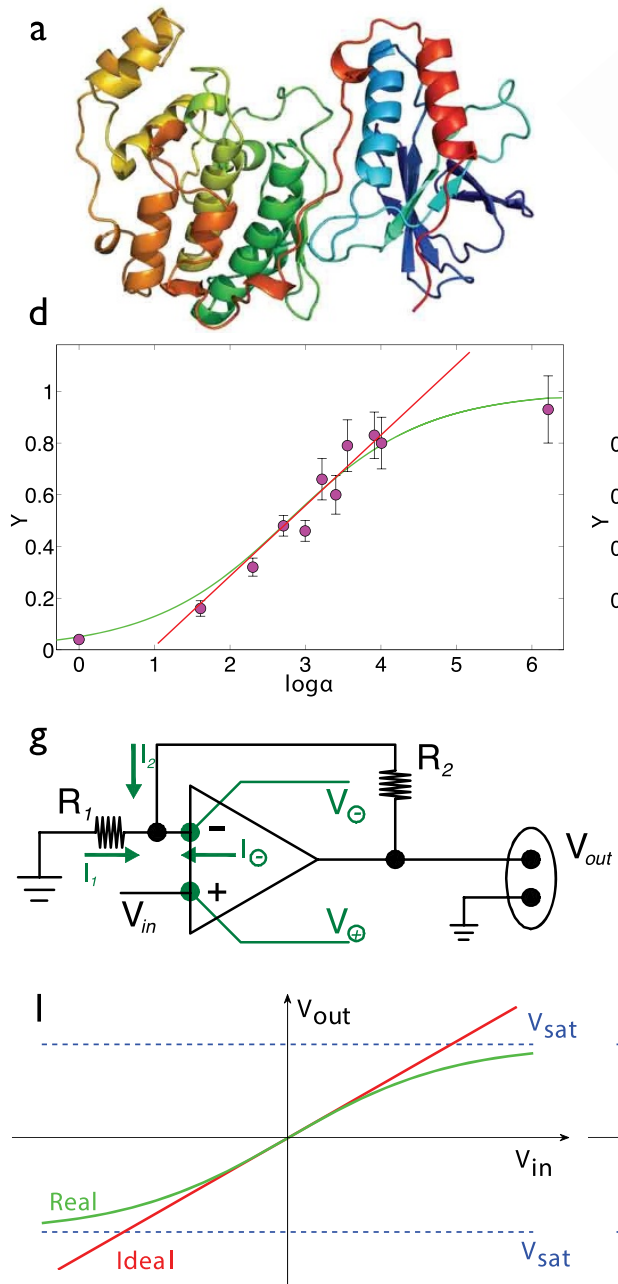

b
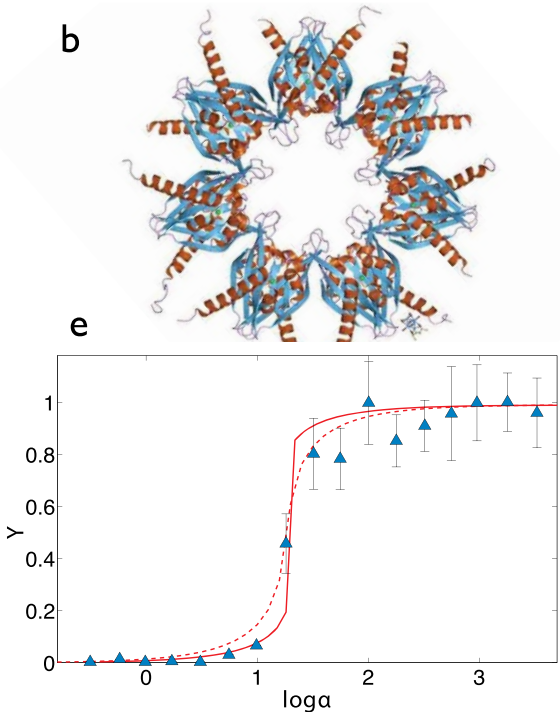

h

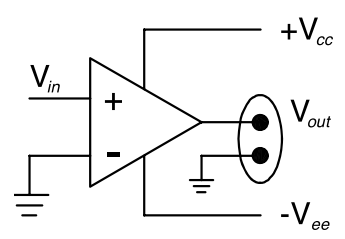

C
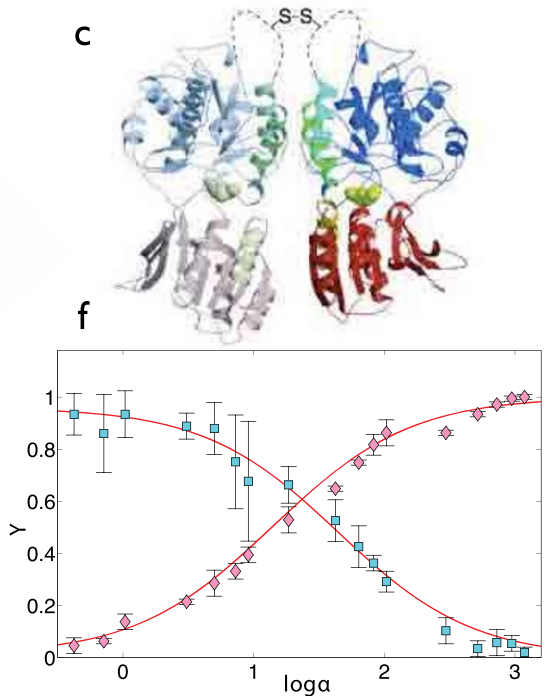

i

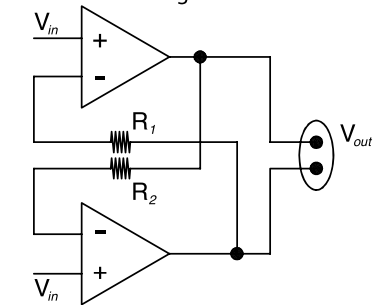

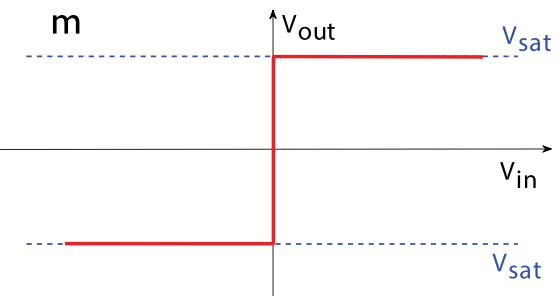

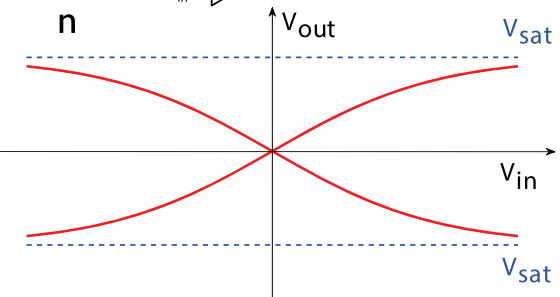

Figure 6 This figure summarizes all the analogies described in the paper: In the first row, pictures of three biological systems exhibiting cooperativity, namely Mitogen-activated protein kinase 14 (positive cooperativity, panel (a), Ca ${ }^{2+}$ calmodulin dependent protein kinases II (ultra-sensitive cooperativity, panel (b), and Synaptic Glutamate receptors (negative cooperativity, panel (c) are shown. The related saturation curves (binding isotherms) are shown in

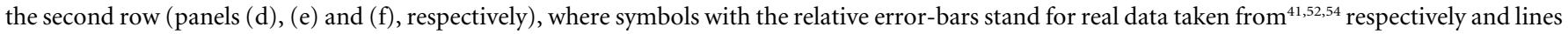
are best fits performed through the analytical expression in eq. 28, obtained from statistical mechanics. The related best-fit parameters are $J=0.14, J=$ $1.16, J=0.29$, respectively. Notice that in panel (d) it is possible to see clearly the "saturation" phenomenon as the first and the last experimental points are far from the linear fit (red line), while are perfectly accounted by the hyperbolic tangent predicted by statistical mechanics (green line), whose correspondence with saturation in electronics is represented in panel (l). Notice further that in panel (e), we compared the ultra-sensitive fit (solid line), with a simple cooperative fit (dashed line): at small substrate concentration the latter case does not match, within its variance, the data points (so accurately measured that error bars are not reported), while the former case is in perfect agreement with data points. In the third row we sketch the cybernetic counterparts, i.e., the operational amplifier (panel (g)), represented as an inverted flip-flop mirroring the symmetry by which we presented the statistical mechanics framework (the standard amplifier is shown in fig. 3), the analog-to-digital converter (panel (h)) and the flip-flop (panel (i)). The (theoretical) transfer functions corresponding to the circuits are finally shown in the fourth row (panels (l), (m) and (n), respectively) for visual comparison with the second one. 